\title{
Automatic real-time collection of RCS of airplanes in a real bistatic low-frequency configuration using a software defined passive radar based on illuminators of opportunity
}

\author{
Jonathan Pisane $^{*, \dagger}$, Sylvain Azarian ${ }^{\dagger}$, Marc Lesturgie $^{\dagger}$, Jacques Verly* \\ *: Dept. of Electricity, Electronics and Computer Science (Montefiore Institute), University of Liège, \\ Grande Traverse 10, B-4000 Liège, Belgium \\ †: SUPELEC / E3S - EA4454 / SONDRA, Rue Joliot-Curie 3, 91192 Gif-sur-Yvette, France
}

Keywords: Multistatic passive radar, RCS collection, cognitive software-defined radio, ATR.

\begin{abstract}
In this paper, a novel method of measurement of the real RCS of various airplanes in real conditions, in bistatic mode, and at low frequency is derived. The presence of an airplane is detected by the use of an ADS-B receiver. The RCS of an airplane is derived thanks to the ratio of the power of the received echo signal to the power of the received direct path signal. In practical experiments, a VOR transmitter is used as illuminator of opportunity, and a software-defined radio is used as receiver. The measured RCS will later be used to classify airplanes, in the context of ATR.
\end{abstract}

\section{INTRODUCTION}

In the context of automatic target recognition (ATR) in particular, and in classification in general, one of the biggest issues is to collect data close enough to reality. Usually, simulated data is used to test the classification algorithms. In a small number of cases, data collected in an anechoic chamber is available. In this case, the acquired data is complete since all configurations can be tested, depending on the capabilities of the anechoic chamber.

In this paper, we present a novel technique of collecting, at rather low cost, real data, in real-time. The realistic assumption made is that the data acquired is reproducible through time, since only a single configuration can be tested at a time. In order to obtain realistic data as complete as measurements that can be acquired in an anechoic chamber, i.e. data at different bistatic angles (and different frequencies), one just needs to increase the measurement period.

The data to be collected is the radar cross-section (RCS)

\footnotetext{
${ }^{1}$ This work is funded by FRIA, the Belgian Fund for Research in Industry and Agriculture.
}

of commercial airplanes (also named targets), in bistatic mode, at low frequency using illuminators of opportunity. The data obtained will be used later to classify the targets at different bistatic angles. From the reproducibility assumption, the collected RCS of a particular type of aircraft during different passes with different configurations leads to the complete characterization of that particular type of target, even though the different passes are not measured on the same airplanes. For example, the target type "A320" can be measured on different flights "AF123" or "SN456".

Section II describes the implemented system that is based on the joint use of an ASD-B receiver, an SDR receiver and a selected illuminator of opportunity. Section III details the different link budgets computed, and their use in the recovery of the RCS of airplanes. Section IV describes the real-time baseband conversion performed by the SDR, allowing to recover the RCS of the targets as described in Section V. A practical example is shown in Section VI. Conclusions and perspectives are drawn in Section VII.

\section{SySTEM IMPLEMENTED}

The system that has to be implemented must complete several requirements. First, the presence of a target has to be detected, and information such as its speed, position, and id number has to be collected. Then, a selected donor transmitter will transmit a continuous and stable (both in power and frequency) signal that will be echoed on the target. This echo signal and the direct-path signal will eventually be recorded and converted into a baseband signal. Those different functions have to be performed in real-time.

Figure 1 shows the system that has been implemented. The presence of a target is detected by the ADS-B receiver, as most commercial airplanes send information on request. This information can be decoded in real-time 
by a commercial ADS-B receiver. If the target is relevant to the system (i.e. the estimated link budget is good enough), the SDR receiver starts recording both the RF signal sent by the selected donor transmitter (also named direct-path signal), and the RF signal transmitted by the selected donor transmitter and echoed on the target (also named the echo signal).

An ideal donor transmitter has to complete several requirements. It must continuously transmits a signal that is constant in power and in frequency, and is band-limited so that the Doppler shift of the target is located outside its frequency content. The ideal transmitted signal $s_{\text {ideal }}(t)$ can be written as

$$
s_{\text {ideal }}(t)=A e^{2 \pi j f t}
$$

The ideal case is nearly reached by using the subcarrier of a VOR (VHF omni-range) station, used by airplanes to locate themselves [1]. The VOR signal is simple, narrowband, and a great number of VOR exists near airports. The transmitted signal $s_{V O R}(t)$ can be written as

$$
s_{V O R}(t)=A e^{j \phi(t)} e^{2 \pi j f t}
$$

where $A$ is the unknown amplitude, $\phi(t)$ the unknown phase, and $f$ the known frequency of the transmitted signal (in the frequency band $[108 ; 118] \mathrm{MHz}$ ). As this phase is unknown, the recovered RCS will be expressed only in terms of amplitude. The phase of the backscattering coefficient of the target will thus not be considered.

\section{SySTEM LINK BUDGETS}

The RCS of airplanes will be recovered thanks to the computation of the different link budgets. The link budgets also help considering a detected target as being relevant or not. Two link budgets are considered: the direct-path link budget, and the echo-path link budget, computed using the bistatic radar equation.

\section{A. Bistatic radar equation}

The bistatic radar equation is defined as [5]

$$
P_{R-\text { Echo }}=\frac{P_{T} G_{T} G_{R} \lambda^{2} \sigma}{(4 \pi)^{3} R_{R}^{2} R_{T}^{2}},
$$

where $P_{R-E c h o}$ is the received power of the echo signal, $P_{T}$ is the power of the transmitted signal, $G_{T}$ the gain of the transmitting antenna, $G_{R}$ the gain of the reception stage, including the reception antenna, $\lambda$ is the wavelength, $\sigma$ the RCS to be computed, $R_{T}$ the distance between the transmitter and the airplane, and $R_{R}$ the distance between the receiver and the airplane.

$R_{T}, R_{R}$ are computed from the target position data given by the ADS-B receiver, $\lambda$ is given by the selection of the donor transmitter, $G_{R}$ is measured by characterizing the SDR receiver, and $P_{R-E c h o}$ is measured at reception. However, $P_{T}$, and $G_{T}$ are unknown, as it is part of the transmitter characteristics. The computation of the directpath link budget will cancel out those unknown terms.

\section{B. Direct-path link budget}

The classical link budget is expressed as [4]

$$
P_{R-D i r}=\frac{P_{T} G_{T} G_{R-D i r}}{L_{F S}}
$$

where $P_{R-D i r}$ is the power of the direct-path received signal, $G_{R-D i r}$ is the gain of the reception stage, and $L_{F S}$ is the free space loss. $G_{R-D i r}$ is different from $G_{R}$ as the receiving antenna is quasi-omnidirectional in the horizontal plane, but not in the vertical plane. Therefore

$$
G_{R}=G_{R-D i r}+G_{R-A n t}
$$

where $G_{R-A n t}$ represents the antenna gain. Since the antenna gain is compensated, $G_{R}=G_{R-D i r}$ in the link budgets.

Equation 4 is valid under this form only if the transmitter and the receiver are visible from each other. As this is not the case here, due to the terrain configuration, Equation 4 is expressed as

$$
P_{R-D i r}=\frac{P_{T} G_{T} G_{R}}{L_{N L O S}},
$$

where $L_{N L O S}$ represents the losses due to the distance between the transmitter and the receiver, in the non-lineof-sight case. Note that $L_{N L O S}$ is at least equal to $L_{F S}$, with $L_{F S}$ expressed as

$$
L_{F S}=32.45+20 \log F+20 \log R_{L}[d B],
$$

where $F[\mathrm{MHz}]$ is the frequency of the transmitter, and $R_{L}$ [km] the line-of-sight distance between the transmitter and the receiver.

$L_{N L O S}$ can thus be expressed as

$$
L_{N L O S}=L_{F S}+L_{G N D}[d B]
$$

where $L_{G N D}$ represents the part of the losses due to the propagation of the signal through the landscape. 


\section{Extraction of the RCS}

By computing the ratio of Equations 3 and 6, using Equation 8, and re-arranging the terms, the RCS can be expressed as

$$
\begin{aligned}
\sigma & =\frac{P_{R-E c h o}}{P_{R-D i r}} \frac{(4 \pi)^{3} R_{T}^{2} R_{R}^{2}}{\lambda^{2}} \frac{1}{L_{N L O S}} \\
& =\frac{P_{R-E c h o}}{P_{R-D i r}} \frac{4 \pi R_{T}^{2} R_{R}^{2}}{R_{L}^{2}} \frac{1}{L_{G N D}}
\end{aligned}
$$

The only unknown parameter is $L_{G N D}$. However, this parameter is almost constant for all detected targets, as it only depends on the direct path which is constant during a single flight path, since the transmitter and the receiver are both fixed. Therefore, the recovered RCS is not the true RCS, but the RCS multiplied by a constant.

\section{REAL-TIME BASEBAND CONVERSION OF RF SIGNALS BY THE SDR RECEIVER}

This Section describes the baseband conversion of the recorded RF signals performed by the SDR receiver ([2], [3]), into the complex baseband in-phase and quadrature components, $I[n]$ and $Q[n]$, respectively. From the $I[n], Q[n]$ digital components, the process to recover the target RCS, based on Equation 9, is detailed.

The RF signals are recorded over a frequency bandwidth $B$ centered on the frequency of the donor transmitter, $f_{c}$, with a bandwidth larger than twice the maximum possible Doppler shift $f_{\text {Doppler-Max }}$ for an aircraft.

$$
B=\left[f_{c}-f_{\text {Doppler-Max }}-\epsilon ; f_{c}+f_{\text {Doppler-Max }}+\epsilon\right],
$$

The RF received signal is constituted of the following components; the band-limited useful signal at frequency $f_{0}$, noise at frequency above $f_{1}(200 \mathrm{MHz}$ here) noted $n_{1}(t)$, noise at frequency below $f_{1}$ but not in the band $110-120 \mathrm{MHz}$ noted $n_{2}(t)$, and noise at frequency between $110-120 \mathrm{MHz}$, noted $n_{3}(t)$. Note than noise does not mean classical noise, but rather any unwanted signal.

$$
s(t)=a(t) \cos \left(2 \pi f_{0} t+\phi(t)\right)+n_{1}(t)+n_{2}(t)+n_{3}(t),
$$

where $a(t)$ is the complex signal envelope, and $\phi(t)$ is an unknown random phase of the desired signal.

Figure 2 shows the block-diagram of the SDR receiver. The input signal $s(t)$ is first filtered by an 8-th order low-pass filter with cutoff frequency $f_{1}=200 \mathrm{MHz}$. The remaining signal is then filtered by a band-pass filter centered on $f_{c}$. The filtered signal can thus be rewritten as

$$
s(t)=a(t) \cos \left(2 \pi f_{0} t+\phi(t)\right)+n_{3}(t),
$$

$s(t)$ is then converted to an intermediate frequency $f_{I F}$. This operation is performed using a square signal generated by a digital programmable oscillator. The resulting signal $z(t)$ of the mixing and the filtering by a band-pass filter centered on $f_{I F}$ is given by

$$
z(t)=\frac{2}{\pi} a(t) \sin \left(2 \pi f_{I F} t+\phi\right)+n^{\prime}(t),
$$

where $n^{\prime}(t)$ denotes the remaining noise components.

To obtain $I(t)$ and $Q(t), z(t)$ is mixed by a signal at frequency $f_{I F}+\Delta f$, and with the same signal having a phase shift of $\pi / 2$, respectively. $\Delta f$ is chosen so that $I(t)$, and $Q(t)$ do lie in the linear part of the filter of the sound card that will be used to digitalize $I(t)$, and $Q(t)$. The baseband signal $I(t)$ is recovered by passing the result of the mixing of $z(t)$ by the carrier at frequency $f_{I F}+\Delta f$ through a low-pass filter. $I(t)$ can thus be written as

$$
I(t)=\frac{4}{\pi^{2}} a(t) \cos (2 \pi \Delta f t+\phi)+n_{I}(t),
$$

Similarly, $Q(t)$ is found to be

$$
Q(t)=\frac{4}{\pi^{2}} a(t) \sin \left(2 \pi \Delta f t+\phi_{q}\right)+n_{Q}(t)
$$

$I(t)$, and $Q(t)$ are then digitized by a computer sound card, and noted $I[n]$, and $Q[n]$.

The fast Fourier transform (FFT) is then applied on a time-domain window of the received signal, giving $S(f)$, where

$$
S(f)=F F T\{I[n]+j Q[n]\}
$$

Figure 3 shows the moving time-domain window for which one $S\left(f, t_{i}\right)$ is obtained for every position $i$ of the window.

The spectrogram $S(f, t)$ will be used to recover, for every target of the database, the target signature, and thus the target RCS at every time instant $t$ of its trajectory.

\section{SignAl POST-PROCESSING USED TO RECOVER THE BISTATIC RCS OF TARGETS}

From the spectrogram $S(f, t)$, it is possible to recover the direct path data, and the target signature along the target trajectory. From the target signature, the RCS can be computed by using Equation 9 . 
The process to recover the target signature is the following. First, notch filters centered on the frequency of the transmitted signal $f_{c}$ and on the frequency of its side-lobe signals, are applied. The power of the direct-path signal at every time instant is recovered. Then, for every time instant, the Doppler shift induced by the target is known, thanks to the smoothed data given by the ADS-B receiver. Thus, at a particular time instant, the maximum of the spectrogram inside a window centered on the frequency of the given Doppler shift corresponds to the target signature at that particular time instant.

The RCS of a target at every time instant of its trajectory is recovered from the value of the target signature by applying Equation 9. This process is applied to every spectrogram recorded in the database, thus allowing to constitute a complete database of RCS of targets.

\section{Practical EXAMPLE}

As an example, the process described above is applied to the measurement of the RCS of a particular airplane, by using a VOR transmitter as illuminator of opportunity that is transmitting a carrier signal at a frequency of 114.7 $\mathrm{MHz}$. Figure 4 shows the spectrogram that has been recorded during the trajectory of a particular aircraft. It can be seen that there are more than one target present during the entire pass. Figure 5 presents the Doppler shift given by the ADS-B data, and its smoothed version. The smoothing is necessary, as the data given by the ADS-B receiver is not exact, due to quantification errors and inconstant times of data transmission.

Figures 6 and 7 show the direct-path and echo-path signal-to-noise ratio, respectively, indicating an SNR of about $30 \mathrm{~dB}$ for the direct-path signal, and an SNR around $15 \mathrm{~dB}$ for the echo-path signal.

Figure 8 shows the computed RCS of the target along its trajectory. The gaps in the RCS plot are either due to the notch filters, to an SNR of the echo-path signal not high enough, or to the non-periodicity of the received ADS-B data. The RCS of the target can be interpolated to fill in the gaps.

Figure 9 shows the variation of the RCS for different A319 airplanes. It can be seen that the reproducibility assumption is realistic, as the RCS presents the same variation from one airplane to the other.

\section{CONCLUSION}

The work presented in this paper validates the possibility of collecting RCS of real targets, in real-time and real conditions, with low investment. The system implemented relies on the use of an SDR receiver used to record both the direct-path signal from the VOR transmitter playing the role of an illuminator of opportunity, and the signal transmitted by the VOR transmitter and echoed on an airplane target that has been detected by an ADS-B receiver. A post-processing stage is needed to recover the target RCS from the spectrogram recorded during its trajectory. The link budgets show that the recovered RCS is the true RCS multiplied by a factor having a small variation from one aircraft trajectory to the other.

Future work includes the classification of targets whose RCS have been recorded by such a system described in this paper. There will be one classification experiment per bistatic angular sector, as the RCS of aircrafts varies according to the bistatic angle.

The system can also be extended to multiple transmitters and receivers, provided a good selection of the transmitters or a wide-band receiving capability. This should provide both spatial and frequency diversity, hence allowing applications such as imaging.

\section{REFERENCES}

[1] United States. Federal Aviation Administration. North Atlantic international general aviation operations manual, volume 4 . The Administration, 1990

[2] P.B. Kenington. RF and baseband techniques for software defined radio. Artech House, 2005.

[3] J. Mitola III and G.Q. Maguire Jr. Cognitive radio: making software radios more personal. Personal Communications, IEEE, 6(4):13-18, 1999.

[4] J.G. Proakis. Digital communications, volume 1221. McGraw-hill, 1987.

[5] N.J. Willis. Bistatic radar. SciTech Publishing, 2005.

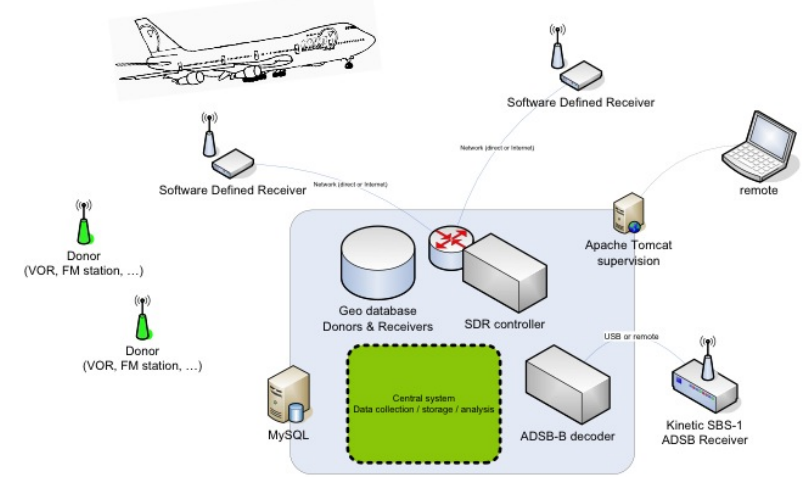

Fig. 1. Architecture of the implemented system. It consists of an aircraft whose presence has been detected by an ADS-B receiver, and of an SDR receiver that records the direct-path signal transmitted by a donor transmitter and the direct-path signal echoed on the airplane. The central system stores the recorded data. 


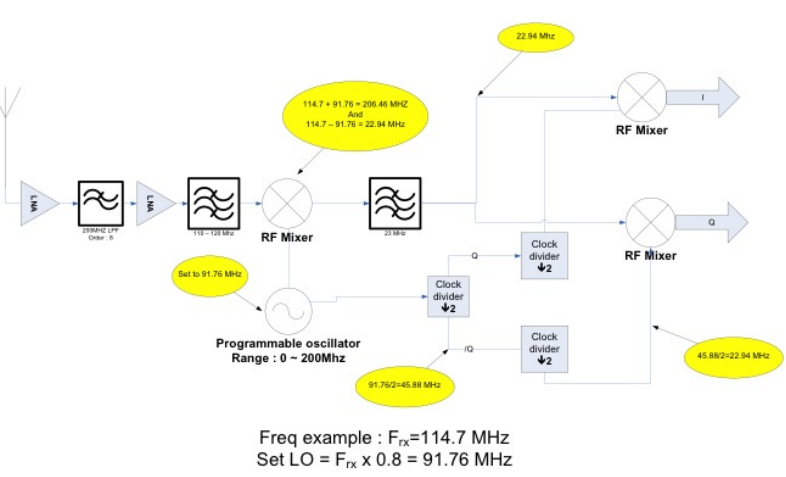

Fig. 2. Conversion of the RF input signals to baseband performed by the SDR receiver. The input signal is first filtered twice and then successively converted from RF to IF and from IF to baseband by a digital mixer, allowing to use only a single oscillator.

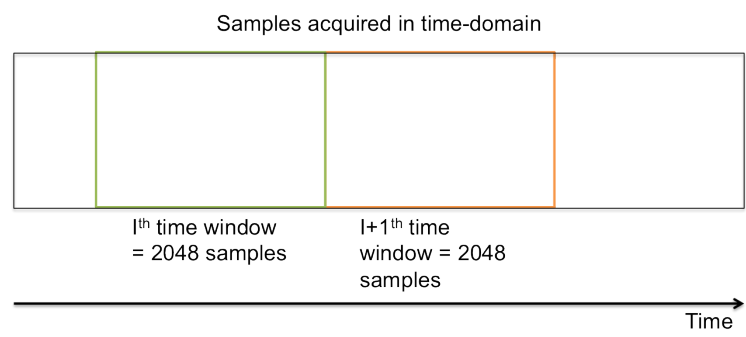

Fig. 3. Integration of the time-domain data in order to obtain the spectrogram $U(f, t)$.

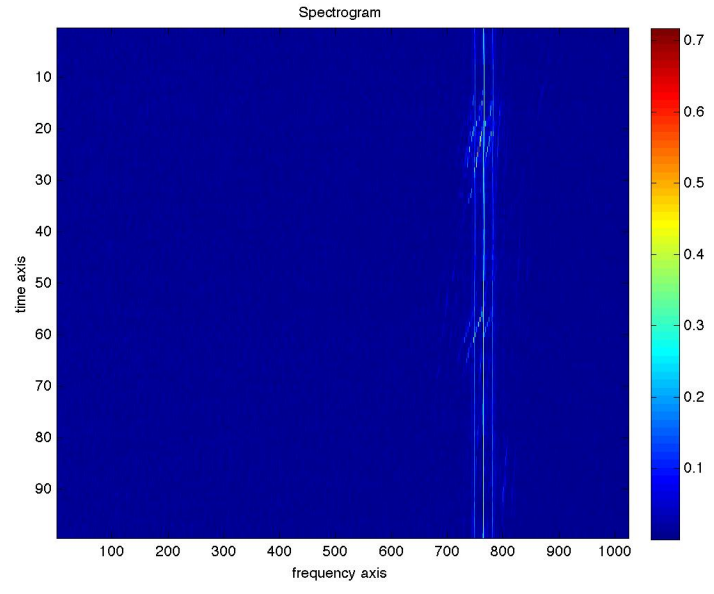

Fig. 4. Spectrogram recorded during the trajectory of a particular airplane. The direct-path signal and its two sidelobes are clearly visible, as well as the signatures of two different airplanes.

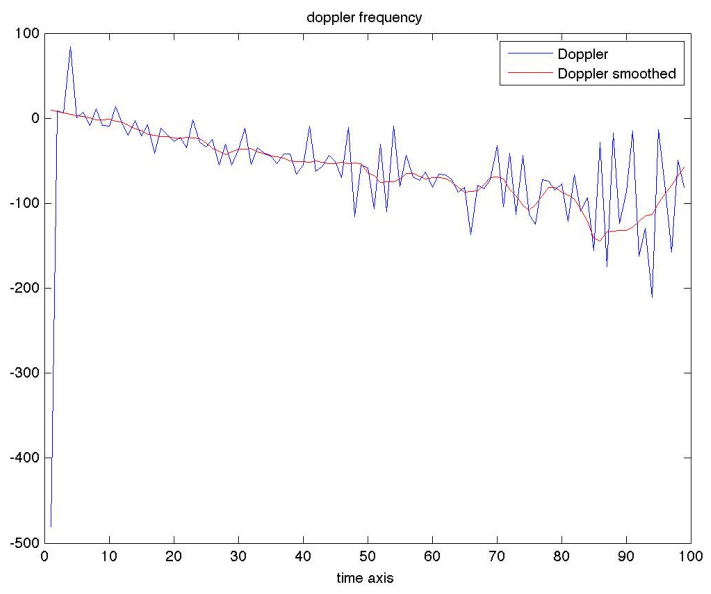

Fig. 5. Doppler shift given by the ADS-B receiver, and its smoothed version.

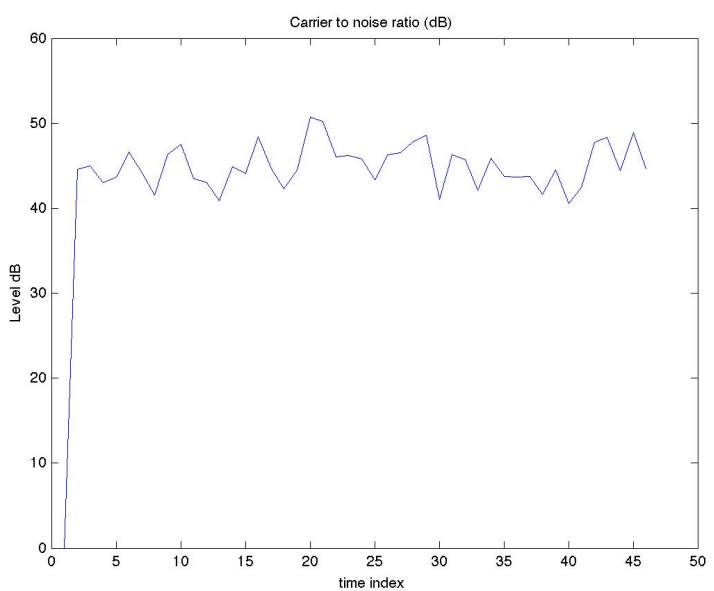

Fig. 6. Signal-to-noise ratio for the direct-path signal. It shows an SNR of at least $30 \mathrm{~dB}$.

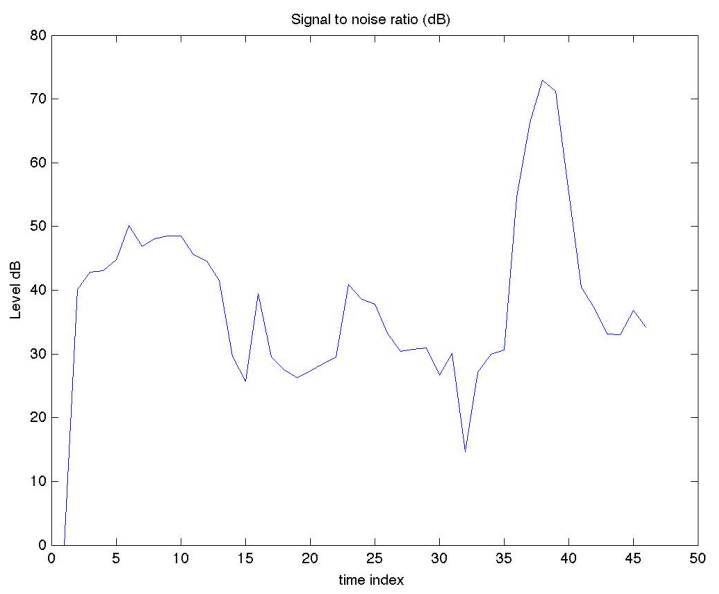

Fig. 7. Signal-to-noise ratio for the echo-path signal. The SNR is about $15 \mathrm{~dB}$ on average. 


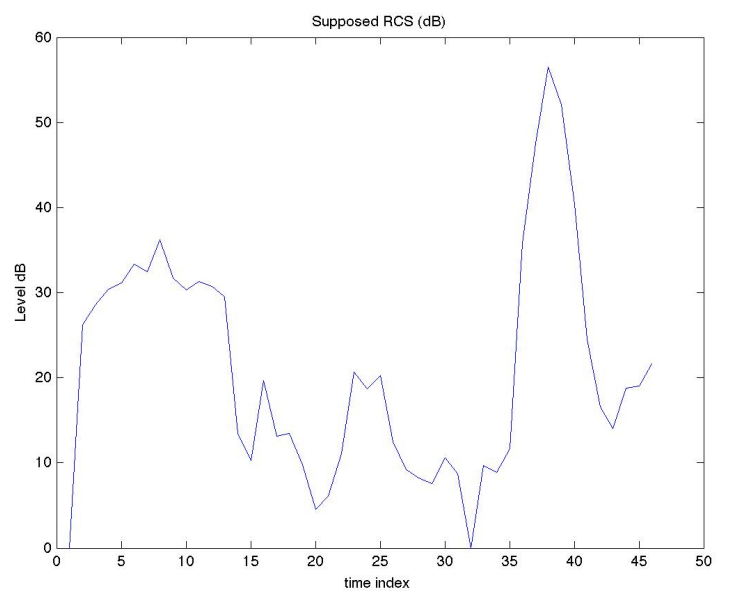

Fig. 8. Computed RCS of an airplane, along its trajectory. The RCS is augmented by the value of the propagation losses.

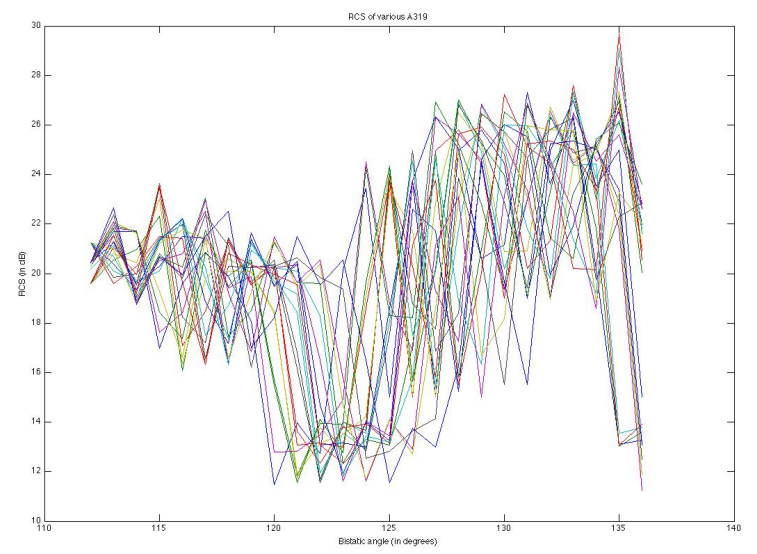

Fig. 9. Computed RCS of A319 airplanes. It can be seen that the RCS is almost the same from one flight to the other, thus asserting the reproducibility assumption. 